\title{
SUBGROUPS OF ALMOST SEPARABLE TORSIONFREE ABELIAN GROUPS
}

\author{
CLAUDIA METELLI
}

(Communicated by Bhama Srinivasan)

ABSTRACT. A characterization is given of those subgroups of an almost separable torsionfree abelian group which inherit separability from the group.

\section{INTRODUCTION}

In this paper we give a fairly general answer to the question "Which pure subgroups of a separable torsionfree abelian group $G$ are separable?". By investigating the splitting properties typical of separability, it was proved classically by Fuchs [FII] that direct summands of $G$ are separable, and recently, by L. G. Nongxa in $[\mathrm{N}]$, that "strongly pure" subgroups of $G$ are separable.

An attempt to deal with the "necessity" side of the question shows that it is too loosely phrased: it is easy to provide examples of completely decomposable pure subgroups of a separable group $G$, having little or nothing to do with what we experience as the "separation structure" of $G$. We thus use our previous investigation of the $t$-local properties typical of separability [M1, M3], to specify the initial question by demanding that the separable subgroups "inherit" their separability from $G$.

To make our request more specific, recall that, for an element $g$ of the separable group $G$, the "separation type" $T_{G}(g)$ of $g$ denotes (what amounts to) the isomorphism class $[X]$ of a minimal rank completely decomposable summand $X$ of $G$ containing $g$. We say that the separable pure subgroup $B$ of $G$ "preserves separation type" if, for each $b \in B, T_{G}(b)=T_{B}(b)$. Note that direct summands, as well as strongly pure subgroups, preserve separation type. The question we consider then becomes: "Which pure subgroups of $G$ are separable with preserved separation type?". To answer it, we deal with the more general class of "almost separable groups" introduced in [M3], and prove that a subgroup $B$ of an almost separable group $G$ is pure, almost separable,

Received by the editors December 12, 1987 and in revised form May 1988.

1980 Mathematics Subject Classification (1985 Revision). Primary 20K20, 20 K27. 
with preserved separation type, if and only if $B$ is a $T T^{*}$-subgroup of $G\left(T T^{*}\right.$ subgroups, defined in [M3], are subgroups which derive their $t$-local structure from $G$ ). An easy reduction then shows that $T T^{*}$-subgroups are the answer in the separable case as well (Theorem 3.2).

As a final by-product, we note that, while being quite different in general, in separable groups $T T^{*}$-subgroups coincide with strongly pure subgroups: so we get the converse of Nongxa's result.

\section{NOTATION AND PRELIMINARY RESULTS}

"Group" means "torsionfree abelian group". Notation and terminology are those of [FII, M1, M3]: we recall here some of it. $B \leq_{*} G$ means that $B$ is a pure subgroup of $G ; B \leq_{\oplus} G$, that $B$ is a direct summand of $G$. If $G=A \oplus B$, we will write $g=a \oplus b$ for the induced direct decomposition of $g \in G$, with no further explanation of the symbols. In the proper context, $n \mid g$ $(n+g)$ means: $n$ divides (does not divide) $g$. For $G=\langle a\rangle_{*} \oplus H$, we say $g \in G$ is substitutable to $a$ in the given decomposition of $G$, if $G=\langle g\rangle_{*} \oplus H$; for $g=a \oplus h$, this holds if and only if $\chi(h) \geq \chi(a)$ [M3, Lemma 0]. $g \in G$ is proper with respect to $B \leq_{*} G$, if $\langle g, B\rangle_{*}=\langle g\rangle_{*} \oplus B ; g$ is extractible in $G$ if $\langle g\rangle_{*} \leq_{\oplus} G$, in which case the type $t(g)$ of $g$ is called an extractible type of $G ; T(G)$ denotes the set of extractible types of $G$.

$\mathrm{T}$ will be the set of all types; for $t^{\prime}, t^{\prime \prime} \in \mathrm{T}, t^{\prime \prime}>_{\text {n.c. }} t^{\prime}$ means $t^{\prime \prime}$ is greater than or noncomparable to $t^{\prime}$; and analogously for $\geq_{\text {n.c. }}$. For $t \in \mathbf{T}$, we consider the functorial pure subgroups of $G: G(t)=\{g \in G \mid t(g) \geq t\}$ and $G^{*}\{t\}=\bigcap_{U \in \mathscr{U}_{1}} U$, where $\mathscr{U}_{t}$ is the family of all pure corank one subgroups of $G$ whose cotype is $\leq t ; G^{*}\{t\}$ is then the reject of $R$ in $G$, where $R$ is a type $t$ subgroup of $\mathbb{Q}$.

We complete the definitions given in [M3] by

Definition 0.1. Let $E$ be a subset of the group $G$. We define $\chi_{G}(E)$, the characteristic of $E$ in $G$, as follows: if $E \neq \varnothing, \chi_{G}(E)=\sup _{g \in E} \chi_{G}(g)$; while $\chi_{G}(\varnothing)=0$, thus adding 0 , as an absolute minimum, to the set of characteristics. We also add $\infty$ as an absolute maximum, and define $\chi_{G}(0)=\infty$. Note that for $g \in G$ we have $\chi_{G}(g)=\chi_{G}(\{g\})$. We say $e \in E$ is a faithful element of $E$ if $\chi_{G}(e)=\chi_{G}(E)$.

As a consequence, $\chi_{G}(E)=\infty$ whenever $0 \in E$; in particular, for any subgroup $E$ of $G$. This definition enables us to deal with cosets $g+H$ of the subgroup $H$ of $G$ : e.g., if $H \leq_{*} G$, then $\chi_{G}(g+H)=\chi_{G / H}(g+H)$, thus simplifying notation; $g$ is proper with respect to $H$ if $g$ is a faithful element of $g+H$.

The newly defined characteristic respects inclusion between arguments as well as between indices, and in fact satisfies the same properties as does the usual characteristic of elements:

Lemma 0.2. If $E, E^{\prime}$ are subset of $G$, then:

(a) if $E \subseteq E^{\prime}$, then $\chi_{G}(E) \leq \chi_{G}\left(E^{\prime}\right)$; 
(b) $\chi_{G}\left(E+E^{\prime}\right) \geq \inf \left(\chi_{G}(E), \chi_{G}\left(E^{\prime}\right)\right)$;

(c) If $\left\langle E, E^{\prime}\right\rangle_{*} \equiv\langle E\rangle_{*} \oplus\left\langle E^{\prime}\right\rangle_{*} \bmod \left(E \cap E^{\prime}\right)_{*}$, then $\chi_{G}\left(E+E^{\prime}\right)=\inf \left(\chi_{G}(E), \chi_{G}\left(E^{\prime}\right)\right)$.

Remark. Definition 0.1 allows a natural definition for a "nice subgroup" of the torsionfree group $G: H \leq G$ is nice if each coset of $H$ in $G$ contains a faithful element.

We recall now from [M3] the definition and principal properties of $T^{*}$ - and $T T^{*}$-subgroups. We will use these results in the following without explicit reference.

A subgroup $B$ of $G$ is a $T^{*}$-subgroup: $B \leq_{T^{*}} G$, if $\forall t \in \mathbb{T}, B$ satisfies

$\left(\mathrm{a}_{1}\right): B^{*}\{t\}=B \cap G^{*}\{t\}$, and

$\left(\mathrm{a}_{2}\right):\left(B+G^{*}\{t\}\right)_{*}=B+G^{*}\{t\}$.

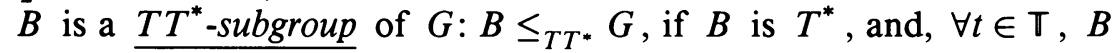
satisfies

$\left(\mathrm{b}_{1}\right): B \cap\left(G(t)+G^{*}\{t\}\right)=B(t)+B^{*}\{t\}$, and

$\left(\mathrm{b}_{2}\right):\left(B+G(t)+\mathrm{G}^{*}\{t\}\right)_{*}=B+\left(G(t)+G^{*}\{t\}\right)_{*}$ [M3, Definition 1.2].

An element $g \in G$ is a $T^{*}$ element of $G$ if $g$ is proper with respect to $G^{*}\{t(g)\}$, i.e. if $\left\langle g, G^{*}\{t(g)\}\right\rangle_{*}=\langle g\rangle_{*} \oplus G^{*}\{t(g)\}$; or, equivalently, if $\langle g\rangle_{*}$ is a $T^{*}$-subgroup of $G$ [M3, Definition 2.6, Prcposition 2.7].

$T^{*}$-subgroups are pure [Observation 1.a: note that the proof given in [M3] is incorrect; the result follows from the fact that, for $\left.q=t(\mathbb{Q}), G^{*}\{q\}=0\right]$; direct summands are $T T^{*}$ [M3, Proposition 1.4], $T^{*}$ and $T T^{*}$ are inductive and transitive.

We use the handiness of Definition 0.1 to sharpen the result of Proposition $1.6 \mathrm{~b}$ in $[\mathrm{M} 3]$.

Proposition 0.3. Let $A \leq B \leq G$. Then $A \leq_{T^{*}} G$ implies $A \leq_{T^{*}} B$, and $A \leq_{T T^{*}} G$ implies $A \leq_{T T^{*}} B$.

Proof. We have $A^{*}\{t\} \leq A \cap B^{*}\{t\} \leq A \cap G^{*}\{t\}=A^{*}\{t\}$, thus $A \leq B$ satisfies $\left(\mathrm{a}_{1}\right)$. To prove $\left(A+B^{*}\{t\}\right) \leq_{*} B$, note that, if $a \in A, a+A^{*}\{t\} \subseteq a+B^{*}\{t\} \subseteq$ $a+G^{*}\{t\}$, therefore $\chi_{A}\left(a+A^{*}\{t\}\right) \leq \chi_{B}\left(a+A^{*}\{t\}\right) \leq \chi_{B}\left(a+B^{*}\{t\}\right) \leq \chi_{G}(a+$ $\left.B^{*}\{t\}\right) \leq \chi_{G}\left(a+G^{*}\{t\}\right)$, which by $\left(\mathrm{a}_{2}\right)$ equals $\chi_{A+G^{*}\{t\}}\left(a+G^{*}\{t\}\right)=\chi_{A}(a+$ $\left.A \cap G^{*}\{t\}\right)=\chi_{A}\left(a+A^{*}\{t\}\right)$ by $\left(\mathrm{a}_{1}\right)$. Hence $\chi_{B}\left(a+B^{*}\{t\}\right)=\chi_{B}\left(a+A^{*}\{t\}\right)=$

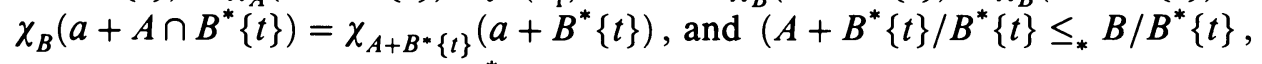
as desired. The proof for $T T^{*}$ runs analogously.

A group $G$ is $t$-bihomogeneous if $G=G(t)$ and $G^{*}\{t\}=0$. If the group $G$ is $t$-bihomogeneous, its pure subgroups are $t$-bihomogeneous and $T T^{*}$ [M3, Lemma 2.2, Corollary 2.4]. A $t$-bihomogeneous subgroup is $T T^{*}$ iff it satisfies $\left(a_{1}\right),\left(a_{2}\right)$ for the type $t$ [M3, Lemma 2.3]. We restate explicitly

Lemma 2.8 [M3]. For a subgroup $X=\bigoplus_{t \in \mathrm{T}} X_{t}$ of $G$, where each $X_{t}$ is $t$ bihomogeneous, the following are equivalent: (i) $X \leq_{T T^{*}} G$; (ii) $X \leq_{T^{*}} G$; (iii) $\forall t \in \mathbb{T}, X_{t} \leq_{T^{*}} G$; (iv) $\forall t \in \mathbb{T}, \forall x \in X_{t}, x$ is a $T^{*}$ element of $G$. 
Finally, we say an element $g \in G$ is separable in $G$ if $g \in X \leq_{\oplus} G$, with $X$ completely decomposable; $g$ is almost separable in $G$ if $g \in X \leq_{T^{*}} G$, with $X$ completely decomposable; a group $G$ is almost separable if every $g \in G$ is almost separable in $G$. We will use

Definition 0.4. For $g \in G$ and $t \in \mathbf{T}$, introduce:

-the lower $t$-characteristic of $g$ in $G: \chi_{t, G}^{\prime}(g)=\chi_{G}\left(g+G^{*}\{t\}\right)$, and

-the upper $t$-characteristic of $g$ in $G: \chi_{t, G}^{\prime \prime}(g)=\chi_{G}\left(g+\left(G(t)+G^{*}\{t\}\right)_{*}\right)$;

- the separation type of $g$ in $G$ is the set $T_{G}(g)=\left\{t \in \mathrm{T} \mid \chi_{t, G}^{\prime}(g)<\right.$ $\left.\chi_{t, G}^{\prime \prime}(g)\right\}$ (as in [M3]; note that $\leq$ always holds). If $B \leq G$, we say the inclusion of $B$ in $G$ preserves separation types if, $\forall b \in B, T_{B}(b)=T_{G}(b)$.

The characterization of the separation type used in the introduction is in Lemma 3.3 [M3]. If $g$ is almost separable in $G$, then $T_{G}(g)=T(X)$, where $X$ is any minimal rank completely decomposable $T^{*}$-subgroup of $G$ containing $g$.

Note that in this case $X$ is short, i.e. $|T(X)|=\operatorname{rk}(X)$.

Lower and upper $t$-characteristics increase along homomorphisms:

Proposition 0.5. Let $G, \bar{G}$ be (torsionfree abelian) groups, and $f: G \rightarrow \bar{G} a$ homomorphism. Then, $\forall g \in G$ and $\forall t \in \mathbf{T}$, we have

$$
\chi_{t, G}^{\prime}(g) \leq \chi_{t, \bar{G}}^{\prime}(f(g)) \text {, and } \chi_{t, G}^{\prime \prime}(g) \leq \chi_{t, \bar{G}}^{\prime \prime}(f(g)) \text {. }
$$

Proof. First, let $f$ be surjective, and w.l.o.g. $\bar{G}=G / K$, where $K=\operatorname{ker} f$. From [M1, Proposition 1.2.c] we get $\left(K+G^{*}\{t\}\right) / K \leq(G / K)^{*}\{t\}$, thus, writing $G^{*}\{K, t\}$ for the preimage, in $G$, of $(G / K)^{*}\{t\}$, and, using Lemma 0.2 above, we have $\chi_{t, G}^{\prime}(g)=\chi_{G}\left(g+G^{*}\{t\}\right) \leq \chi_{G}\left(g+K+G^{*}\{t\}\right) \leq \chi_{G}\left(g+G^{*}\{K, t\}\right)=$ $\chi_{G / K}\left(g+K+(G / K)^{*}\{t\}\right)=\chi_{t, \bar{G}}^{\prime}(f(g))$. The proof for $\chi^{\prime \prime}$ runs analogously, since

$$
\frac{K+\left(G(t)+G^{*}\{t\}\right)_{*}}{K} \leq\left(\frac{K+G(t)}{K}+\frac{K+G^{*}\{t\}}{K}\right)_{*} \leq\left(\frac{G}{K}(t)+\frac{G}{K}^{*}\{t\}\right)_{*},
$$

therefore

$$
\begin{aligned}
\chi_{t, G}^{\prime \prime}(g) & =\chi_{G}\left(g+\left(G(t)+G^{*}\{t\}\right)_{*}\right) \leq \chi_{G}\left(g+K+\left(G(t)+G^{*}\{t\}\right)_{*}\right) \\
& \leq \chi_{G / K}\left(g+K+\left((G / K)(t)+(G / K)^{*}\{t\}\right)_{*}\right)=\chi_{t, \bar{G}}^{\prime \prime}(f(g)) .
\end{aligned}
$$

We complete the proof if we show that, whenever $h \in H \leq G, \chi_{t, H}^{\prime}(h) \leq$ $\chi_{t, G}^{\prime}(h), \chi_{t, H}^{\prime \prime}(h) \leq \chi_{t, G}^{\prime \prime}(h)$. From [M1, Proposition 1.2.b] we have $H^{*}\{t\} \leq$ $H \cap G^{*}\{t\}$, thus $\chi_{t, H}^{\prime}(h)=\chi_{H}\left(h+H^{*}\{t\}\right) \leq \chi_{H}\left(h+H \cap G^{*}\{t\}\right)=\chi_{H+G^{*}}\{t\}$ $\left(h+G^{*}\{t\}\right) \leq \chi_{G}\left(h+G^{*}\{t\}\right)=\chi_{t, G}^{\prime}(h)$. The proof for $\chi^{\prime \prime}$ runs analogously, since $\left(H(t)+H^{*}\{t\}\right)_{*} \leq\left(G(t)+G^{*}\{t\}\right)_{*}$. 


\section{Preserving SeParation types}

We prove now that, in looking for subgroups $B$ of an almost separable group $G$ which are almost separable with preserved separation type (see Definition 0.4 ), we may restrict ourselves to $T^{*}$-subgroups of $G$; and similarly for the separable case.

Proposition 1.1. Let $G$ be almost separable, $B$ an almost separable pure subgroup of $G$. Then the following are equivalent:

(i) The inclusion of $B$ in $G$ preserves separation types (i.e. $\forall b \in B$, $\left.T_{B}(b)=T_{G}(b)\right)$

(ii) $B \leq_{T^{*}} G$.

Proof. (i) $\Rightarrow$ (ii) will be proved by contradiction. Suppose $B$ is not $T^{*}$ in $G$ because $\left(\mathrm{a}_{1}\right)$ does not hold, i.e. there is $b \in B \cap G^{*}\{t\} \backslash B^{*}\{t\}$. Then $\chi_{t, G}^{\prime}(b)=$ $\infty$, thus for each type $t^{\prime} \leq t, t^{\prime} \notin T_{G}(b)$; since $B$ is almost separable, $b \in$ $Y \leq_{T^{*}} B, Y$ completely decomposable; $b \notin B^{*}\{t\}$, thus $b \notin Y^{*}\{t\}$, hence $T_{Y}(b) \ni t^{\prime} \leq t$, thus $T_{Y}(b)=T_{B}(b) \neq T_{G}(b)$, against (ii).

Assume next $\left(\mathrm{a}_{1}\right)$ holds, but $\left(\mathrm{a}_{2}\right)$ fails. Then there is $b \in B \backslash B^{*}\{t\}$ and $n \in \mathbb{N}$ such that $n \mid\left(b+G^{*}\{t\}\right)$ in $G / G^{*}\{t\}$ but not in $\left(B+G^{*}\{t\}\right) / G^{*}\{t\} \cong$ $B / B^{*}\{t\}$; thus $\chi_{t, G}^{\prime}(b)>\chi_{t, B}^{\prime}(b)$. Let now $B$ be almost separable, $b=b^{\prime} \oplus$ $b^{*}$, with $b^{*} \in B^{*}\{t\}$, and $b^{\prime} \in Y^{\prime}$, a completely decomposable $T^{*}$-subgroup of $B$ such that $Y^{\prime} \cap B^{*}\{t\}=0$ (so $T_{B}\left(b^{\prime}\right)$ consists of types $\left.\leq t\right)$. Then $n \mid\left(b+G^{*}\{t\}\right)=b^{\prime}+G^{*}\{t\}$ in $G / G^{*}\{t\}$, but $n \nmid\left(b+B^{*}\{t\}\right)=b^{\prime}+B^{*}\{t\}$ in $B / B^{*}\{t\}$, thus $n \mid b^{\prime}$ (in $B$, hence in $G$ ). If $G$ is almost separable, write analogously $b^{\prime}=x^{\prime} \oplus x^{*}$, where $x^{*} \in G^{*}\{t\}$, and $x^{\prime} \in X^{\prime}$, a completely decomposable $T^{*}$-subgroup of $G$ with $X^{\prime} \cap G^{*}\{t\}=0$. We have $n+b^{\prime}$, but $n \mid\left(b^{\prime}+G^{*}\{t\}\right)=x^{\prime}+G^{*}\{t\}$, thus $n \mid x^{\prime}$. Hence $x^{*} \neq 0$, and $T_{G}\left(b^{\prime}\right)$ contains types $>_{\text {n.c. }} t$, thus is different from $T_{B}\left(b^{\prime}\right)$, in contradiction to (i).

(ii) $\Rightarrow$ (i) follows easily from the more general observation that, if $B$ and $G$ are any groups with $B \leq_{T^{*}} G$, and if $b$ is almost separable in $B$, then, for $Y$ completely decomposable such that $b \in Y \leq_{T^{*}} B \leq_{T^{*}} G$, we have $T_{B}(b)=T_{Y}(b)=T_{G}(b)$, as required.

Similar conclusions hold for separable groups, in view of the next lemma.

Lemma 1.2. (i) Let $b$ be an almost separable element of $B \leq_{T^{*}} G$, where $G$ is separable. Then $b$ is separable in $B$. Thus

(ii) Almost separable $T^{*}$-subgroups of separable groups are separable.

Proof. Let $b \in Y \leq_{T^{*}} B \leq_{T^{*}} G, Y$ finite rank completely decomposable. Then $Y \leq_{T^{*}} G$, thus $Y \leq_{T T^{*}} G$, hence by [M3, Proposition 2.9] $Y \leq_{\oplus} G$, thus $Y \leq_{\oplus} B$.

Corollary 1.3. Let $G$ be separable, $B$ a separable pure subgroup $G$. Then the following are equivalent:

(i) The inclusion of $B$ in $G$ preserves separation types;

(ii) $B \leq_{T^{*}} G$. 


\section{2. $T T^{*}$-SUBGROUPS OF ALMOST SEPARABLE GROUPS}

We investigate now the $T^{*}$-subgroups of almost separable groups $G$, to discover which ones are almost separable.

Lemma 2.1. Let $G$ be almost separable. Then $\forall t, \tau \in \mathbb{T}, G(t) \cap G^{*}\{\tau\}=$ $G(t)^{*}\{\tau\}$.

Proof. One inclusion is always true. Let then $g \in G(t) \cap G^{*}\{\tau\}$ : we have $g \in X \leq_{T^{*}} G$, and we may choose $T(X)$ to consist of types $\geq t,>_{\text {n.c. }} \tau$. Any $U \leq_{*} G(t)$ of corank 1 and cotype $\leq \tau$ will then contain $X$, hence $g$ : thus, $g \in G(t)^{*}\{\tau\}$.

Proposition 2.2. Let $G$ be almost separable, $B \leq_{T^{*}} G$. The following are equivalent:

(i) $B$ is almost separable;

(ii) $\forall t \in \mathbb{T}, B$ satisfies $\left(\mathrm{b}_{1}\right): B \cap\left(G(t)+G^{*}\{t\}\right)=B(t)+B^{*}\{t\}$.

Proof. (i) $\Rightarrow$ (ii). Let $B$ be almost separable, and $b \in B \cap\left(G(t)+G^{*}\{t\}\right)$. If $b \in G^{*}\{t\}$, we are done by $\left(\mathrm{a}_{1}\right)$. If not, then any minimal rank completely decomposable $X \leq_{T^{*}} G$ containing $b$ is contained in $\left(G(t)+G^{*}\{t\}\right) \backslash G^{*}\{t\}$ : thus $T_{G}(b)=T(X)=\{t\} \cup T^{\prime}$, where the types of $T^{\prime}$ are $>_{\text {n.c. }} t$. Since $B$ is almost separable, we can choose $Y$ completely decomposable of minimal rank such that $b \in Y \leq_{T^{*}} B$, thus $T_{B}(b)=T_{Y}(b)=T(Y) . \quad Y$ is a $T^{*}$-subgroup of $G$ as well, hence $T_{Y}(b)=T_{G}(b)=\{t\} \cup T^{\prime}$. Then $Y=\langle y\rangle_{*} \oplus Y^{*}$, with $Y^{*} \leq B^{*}\{t\}$, and $b=y \oplus y^{*} \in B(t)+B^{*}\{t\}$, as desired.

To prove (ii) $\Rightarrow$ (i), let $b \in B, b \in X$, a short completely decomposable $T^{*}$-subgroup of $G ;\left|T_{G}(b)\right|=|T(X)|=n$. We show $b$ is almost separable in $B$, by induction on $n$. If $n=1$, by Proposition $0.3 b$ is a $T^{*}$ element of $B$, hence almost separable in $B$. Let $n>1$ and $t$ minimal in $T(X)$; write $b=x \oplus x^{*} \in\langle x\rangle_{*} \oplus G^{*}\{t\}$. It will be enough to prove that $x$ is substitutable, in this decomposition, by a $T^{*}$ element $b^{\prime} \in B$ : for then, $b=b^{\prime} \oplus y^{*}$ with $y^{*} \in G^{*}\{t\}$ yields $y^{*} \in B^{*}\{t\}$, and $\left|T_{G}\left(y^{*}\right)\right|=\left|T_{G}(b) \backslash\{t\}\right|=n-1$; so, by induction hypothesis, $y^{*}$ is almost separable in $B$, and since the separation types of $y^{*}$ and of $b^{\prime}$ are disjoint, $b=b^{\prime}+y^{*}$ is almost separable in $B$ [M3, Lemma 2.8].

Since $b \in B \cap\left(G(t)+G^{*}\{t\}\right)=B(t)+B^{*}\{t\}$, we can write $b=b_{t}+b^{*}$, $b_{t} \notin G^{*}\{t\}$, so that $b_{t}-x=x^{*}-b^{*}=g^{*} \in G(t) \cap G^{*}\{t\}$; we have then $b_{t}=x+g^{*} \in H=B \cap\left(\langle x\rangle_{*} \oplus\left(G(t) \cap G^{*}\{t\}\right)\right) \leq_{*} B \cap G(t)=B(t)$. Now consider

$$
\frac{H}{B \cap G(t) \cap G^{*}\{t\}} \simeq \frac{H+\left(G(t) \cap G^{*}\{t\}\right)}{G(t) \cap G^{*}\{t\}} \leq \frac{\langle x\rangle_{*} \oplus\left(G(t) \cap G^{*}\{t\}\right)}{G(t) \cap G^{*}\{t\}} \simeq\langle x\rangle_{*} .
$$

The left-hand quotient has rank $\leq 1$, type $\leq t$; but $b_{t} \in H \backslash B \cap G(t) \cap G^{*}\{t\}$; thus, $B \cap G(t) \cap G^{*}\{t\}$ is a corank 1, cotype $t$ pure subgroup of $H=H(t)$, 
hence it splits: $H=\left\langle b^{\prime}\right\rangle_{*} \oplus B \cap G(t) \cap G^{*}\{t\}$. Now $b^{\prime}$ is a type $t T^{*}$ element of $B(t)$, since $H \leq_{*} G$ and $B \cap G(t) \cap G^{*}\{t\}=B(t) \cap B^{*}\{t\} \geq B(t)^{*}\{t\}$; moreover, $b_{t}=b^{\prime} \oplus b^{* *}$, with $b^{* *} \in B(t) \cap B^{*}\{t\}$; thus $b^{\prime}=x \oplus\left(g^{*}-b^{* *}\right) \in x+G^{*}\{t\}$, hence $\chi_{G}\left(b^{\prime}\right) \leq \chi_{G}(x)$.

If we show now that $\chi_{G}\left(b^{\prime}\right) \geq \chi_{G}(x)$, we prove that $b^{\prime}$ is substitutable to $x$ in $\langle x\rangle_{*} \oplus G^{*}\{t\}$, and at the same time that $b^{\prime}$ is a $T^{*}$ element of $G$ : for then $t\left(b^{\prime}\right)=t(x)=t$, and $\left\langle b^{\prime}, G^{*}\{t\}\right\rangle_{*}$ (which is equal to $\langle x\rangle_{*} \oplus G^{*}\{t\}$ ) will equal $\left\langle b^{\prime}\right\rangle_{*} \oplus G^{*}\{t\}$.

We have $x=b^{\prime}+\left(b^{* *}-g^{*}\right)$, with $b^{\prime} \in G(t), b^{* *}-g^{*} \in G(t) \cap G^{*}\{t\}=$ $G(t)^{*}\{t\}$ by Lemma 2.1; we get our inequality if we prove that the decomposition of $x$ is direct; and we will get this by showing that $b^{\prime}$ is a $T^{*}$ element of $G(t)$. We know that $\left\langle b^{\prime}\right\rangle_{*} \oplus B \cap G(t) \cap G^{*}\{t\}$ is pure in $B(t)$, thus, via the natural isomorphism, $\left\langle b^{\prime}\right\rangle_{*} \oplus G(t) \cap G^{*}\{t\} \leq_{*} B(t)+G(t) \cap G^{*}\{t\}$. We prove that this last group is pure in $G(t)$, by showing that it is equal to $G(t) \cap\left(B+G^{*}\{t\}\right)$. One inclusion is obvious. Let then $g_{t}=b^{\prime \prime}+g^{*} \in G(t) \cap\left(B+G^{*}\{t\}\right)$ : we have $b^{\prime \prime}=g_{t}-g^{*} \in B \cap\left(G(t)+G^{*}\{t\}\right)=B(t)+B^{*}\{t\}$, thus $b^{\prime \prime}=b_{t t}+b^{*}, b^{*}+g^{*}=$ $g_{t}-b_{t t} \in G(t) \cap G^{*}\{t\}$; therefore, $g_{t}=b_{t t}+\left(b^{*}+g^{*}\right) \in B(t)+G(t) \cap G^{*}\{t\}$. This shows $\left\langle b^{\prime}\right\rangle_{*} \oplus G(t)^{*}\{t\} \leq_{*} G(t)$, as desired.

We complete our result by

Theorem 2.3. Let $G$ be almost separable. For $B \leq_{T^{*}} G$, the following are equivalent:

(i) $B \leq_{T T^{*}} G$;

(ii) $B$ satisfies $\left(\mathrm{b}_{1}\right)$;

(iii) $B$ is almost separable.

Proof. (i) $\Rightarrow$ (ii) holds by definition, while (ii) $\Rightarrow$ (iii) follows from Proposition 2.2. To prove (iii) $\Rightarrow$ (i), we must show $B$ satisfies $\left(b_{1}\right),\left(b_{2}\right)$ for each type $t$.

$\left(\mathrm{b}_{1}\right)$ follows from Proposition 2.2.

$\left(\mathrm{b}_{2}\right)$ To show $H=B+\left(G(t)+G^{*}\{t\}\right)_{*} \leq_{*} G$, let $n \mid h=b+g \in H$, where $b \in B, g \in\left(G(t)+G^{*}\{t\}\right)_{*}$. As before, w.l.o.g. $b \in Y^{\prime}$, a completely decomposable $T^{*}$-subgroup of $B$, with $T\left(Y^{\prime}\right)$ consisting of types $<t$. For $t^{\prime}$ maximal in $T_{G}(b)$, we have $Y^{\prime} \cap G^{*}\left\{t^{\prime}\right\}=0, G^{*}\left\{t^{\prime}\right\} \geq\left(G(t)+G^{*}\{t\}\right)_{*} \ni g$, $Y^{\prime} \oplus G^{*}\left\{t^{\prime}\right\} \leq_{*} G$; thus $h=b \oplus g$ is direct, hence $b=n b^{\prime}$ with $b^{\prime \prime} \in Y^{\prime} \leq B$, $g=n g^{\prime}$ with $g^{\prime} \in\left(G(t)+G^{*}\{t\}\right)_{*}$, as desired.

Corollary 2.4. $T T^{*}$-subgroups of separable groups are separable. In fact, let $G$ be separable: for $B \leq_{T^{*}} G$, the following are equivalent:

(i) $B \leq_{T T^{*}} G$;

(ii) $B$ satisfies $\left(\mathrm{b}_{1}\right)$;

(iii) $B$ is separable. 
Proof. Since separable groups are almost separable, we only need to show (ii) $\Rightarrow$ (iii). This follows from Theorem 2.3 and Lemma 1.2.

\section{CONCLUSIONS}

The results of Paragraphs 1 and 2 can now be summed up to answer our initial question: "Which pure subgroups of a separable group $G$ inherit separability from $G$ ?"

Theorem 3.1. (i) If $G$ is almost separable, the pure almost separable subgroups of $G$ with preserved separation type are exactly the $T T^{*}$-subgroups of $G$.

(ii) If $G$ is separable, the pure separable subgroups of $G$ with preserved separation type are exactly the $T T^{*}$-subgroups of $G$.

Proof. (i) follows from Theorem 2.3, Proposition 1.1; (ii) from Corollary 2.4, Corollary 1.3.

In [N], L. G. Nongxa proved that strongly pure subgroups of separable groups are separable. Theorem 3.1 allows us to characterize the strongly pure subgroups of separable groups, via the following observation:

Proposition 3.2. A subgroup of a separable group is strongly pure if and only if it is a $T T^{*}$-subgroup.

Proof. From the proof of Theorem 4 in [N], it is easy to derive that strongly pure subgroups of separable groups (which are proved there to be separable) preserve separation type: thus, by our last theorem, they are $T T^{*}$-subgroups. On the other hand, a $T T^{*}$-subgroup $B$ of a separable group $G$ is strongly pure: for then, by Corollary $2.4, B$ is separable; by the proof of Lemma 1.2 (i), if $Y$ is a finite rank completely decomposable summand of $B$ containing $b, Y \leq_{\oplus} G$; and since any projection $\pi_{Y}: G \rightarrow Y$ yields a morphism of $G$ in $B$ sending $b$ onto itself, $B$ is strongly pure.

We thus get the converse of Nongxa's result:

Corollary 3.3. A subgroup of a separable group is strongly pure if and only if it is pure, separable, with preserved separation type.

Let us finally note that, if the group $G$ is not separable, the two notions of "strongly pure subgroup" and " $T T^{*}$-subgroup" of $G$ do not coincide. In particular, there are easy examples of $T T^{*}$-subgroups of almost separable groups, which are not strongly pure: for instance, in a bihomogeneous group any finite rank pure subgroup is $T T^{*}$, while only those which are direct summands are strongly pure.

\section{REFERENCES}

[FII] L. Fuchs, Infinite Abelian Groups II, Academic Press, London-New York, 1973.

[M1] C. Metelli, On type-related properties of torsionfree abelian groups, Abelian Group Theory, Proc. Honolulu Conference 1982-1983, Lecture Notes in Math., vol. 1006, Springer, Berlin, 1984, pp. 253-267. 
[M3] _ _ Almost separable torsionfree abelian groups, Abelian Group Theory, Proc. Oberwolfach Conference, 1985, Gordon and Breach, 1987, pp. 275-289.

[N] L. G. Nongxa, Strongly pure subgroups of separable torsion-free abelian groups, Trans. Amer. Math. Soc. 290 (1985), 363-373.

Dipartimento di Matematica Pura e Applicata, via Belzoni 7, 35131 Padova, Italy 